\title{
Internet-delivered cognitive behavioural therapy in the treatment of psychiatric illness
}

\author{
David Gratzer MD, Faiza Khalid-Khan MSW RSW
}

$\mathrm{T}$ he effectiveness of cognitive behavioural therapy (CBT) is well established for many mental illnesses, including mood and anxiety disorders. CBT, which takes into account how thoughts affect emotions and behaviour, has been shown to be as effective for mild and moderate depression as medications. According to the depression guidelines of the Canadian Network for Mood and Anxiety Treatments, the combination of psychopharmacology and CBT is superior to either modality alone. ${ }^{1}$ However, CBT is resource-intensive and consequently is not available to many patients.

Technology may be able to improve access by allowing delivery of CBT via the Internet. We reviewed the evidence for the effectiveness of Internet-delivered CBT and discuss here its applications, advantages and disadvantages, as well as potential future clinical directions for the delivery of this treatment modality. Internet-delivered CBT has been developed and tested in different forms for many common psychiatric diseases, including obsessive-compulsive disorder, post-traumatic stress disorder, and depression and anxiety disorders, both separately and together. The evidence for this review comprises mainly randomized trials and meta-analyses of intervention studies (Box 1).

\section{What is Internet-delivered CBT?}

Internet-delivered CBT is psychotherapy based on CBT principles, delivered via the Internet by an individual or program remote from the client. CBT is based on Beck's Cognitive Triad (people's thoughts, emotions and behaviours influence each other; patients cannot control the world, but they can take control of how they interpret things in that world). CBT isolates specific problems, and a therapist helps the patient to develop strategies to address those problems by changing thinking patterns and behaviours. ${ }^{2}$ CBT has self-contained modules and clearly defined goals; it can be scripted and requires minimal therapist intervention. ${ }^{3}$ Thus, CBT is uniquely suited for implementation online. There are various types of Internet-delivered CBT programs; however, they share many features, being made up of short-term, patient-guided, goal- oriented sessions, ${ }^{4}$ typically consisting of 8 to 12 modules. ${ }^{5}$ They can be made available online or via a smartphone or tablet application (app), free or for a fee, and with or without therapist support (Box 2). Their content and delivery may differ according to the condition being treated.

\section{What is the evidence for Internet- delivered CBT?}

A systematic review of studies published between 2000 and 2012 identified 1104 articles reporting Internet-delivered or computerized treatments for common psychiatric disorders. ${ }^{6}$ Many of the early studies were not rigorously designed. ${ }^{7}$ Recently, the quality of studies has improved, and a more reliable body of literature has developed. Randomized controlled trials (RCTs) investigating Internet-delivered CBT differ with respect to the type of control group, the degree of therapist involvement, the types of communication and the format of the online modules, which makes it difficult to compare their findings. ${ }^{8,9}$ In addition to evidence from these RCTs, we also incorporate literature on smartphone and tablet apps.

We focus here on three conditions - anxiety, depression and emotional distress associated with physical illness - and consider special populations and long-term treatment effectiveness.

\section{Anxiety}

Internet-delivered CBT for anxiety has been evaluated in numerous RCTs and has thus been the subject of several meta-analyses. A 2009 meta-analysis evaluated 19 Internet- or computer-based CBT trials $(n=1167)$ for depression and anxiety. ${ }^{8}$ The length of treatment and post-treatment evaluation

\section{- Ker POINTS}

- Internet-delivered cognitive behavioural therapy (CBT) allows easier and quicker access to CBT with lower cost and greater convenience than traditional therapy.

- Strong evidence from randomized controlled trials indicates that Internet-delivered CBT can be used in clinical practice for many patients.

- Additional studies should include larger sample sizes, with stratification according to diagnosis and level of therapist support.
Competing interests: None declared.

This article has been peer reviewed.

Correspondence to: David Gratzer, dgratzer@me.com

CMAJ 2016. DOI: 10.1503 /cmaj.150007 
times varied among the studies included. The measures evaluated also differed substantially, but could be broadly classified into five categories: depression, anxiety, general distress, dysfunctional thinking, and functioning or quality of life. The effect size ${ }^{10}$ was calculated for each set of measures for all studies and individually for the four diagnostic groups: any anxiety disorder, posttraumatic stress disorder, panic disorder and phobia. After pooling the data, the authors found that patients who received Internet-delivered CBT had fewer symptoms in all clinical measures than did wait-listed controls, with effect sizes ranging from 0.48 to 1.14 (95\% confidence interval [CI] 0.24 to 0.72 and 0.43 to 1.85 , respectively). For comparisons of Internet-delivered CBT with placebo, the effect sizes ranged from 0.49 to 0.88 (95\% CI 0.14 to 0.84 and 0.70 to 1.31 , respectively). Reductions in symptoms with Internet-delivered CBT were equivalent to or better than in-person CBT (i.e., treatment as usual), with mean effect sizes ranging from -0.02 to 0.57 ( $95 \% \mathrm{CI}-0.33$ to 0.30 and 0.22 to 0.92 , respectively). With regard to comparisons with wait-listed controls for the individual diagnostic groups, the mean effect sizes were 0.75 (95\% CI 0.49 to 1.01 ) for post-traumatic stress disorder, 1.20 (95\% CI 0.87 to 1.58$)$ for panic disorder and 0.66 (95\% CI 0.30 to 1.02 ) for phobia. For

\section{Box 1: Evidence used in this review}

We searched PubMed using the search terms "Internet" and "cognitive behavioural therapy" (CBT). We chose the most relevant articles presenting results from randomized controlled trials, meta-analyses and reviews. The quality of the studies varied. We identified many well-controlled, randomized trials of Internet-delivered CBT for various psychiatric conditions. Adherence rates varied, with some studies reporting results for all patients whether they dropped out prematurely or not, and other studies reporting results only for those who completed the program. This discrepancy may dramatically skew effects among studies. In addition, most meta-analyses showed mixed results from studies using Internet-delivered CBT alone and those using other types of computer-assisted CBT. Finally, some meta-analyses mixed studies that included some level of therapist support with studies not including such support. Overall, the evidence supporting integration of Internet-delivered CBT into clinical practice was strong for specific conditions and specific populations.

We also searched the Internet using Google and the search terms "cognitive behavioural therapy," "cognitive behavioral therapy" and "app" to identify the most common apps.

Box 2: Models for using Internet-delivered cognitive behavioural therapy (CBT) in clinical practice

\begin{tabular}{ll}
$\begin{array}{l}\text { Level of therapist } \\
\text { involvement }\end{array}$ & \multicolumn{1}{c}{$\begin{array}{c}\text { Description } \\
\text { Low }\end{array}$} \\
\hline Medium & $\begin{array}{l}\text { Patients are told about websites } \\
\text { Pollowts are given self-directed exercises with some }\end{array}$ \\
\hline High & $\begin{array}{l}\text { Internet-delivered CBT is included in the treatment } \\
\text { plan, with Internet-assisted follow-up and } \\
\text { discussion (e.g., online chats, forums, email) }\end{array}$ \\
\hline
\end{tabular}

comparisons with placebo control, the mean effect sizes were 0.93 (95\% CI 0.49 to 1.38 ) for panic disorder and 0.81 (95\% CI 0.44 to 1.19$)$ for phobia. In addition, for comparison of Internetdelivered CBT with treatment as usual for panic disorder, the outcomes were comparable (mean effect size $0.30,95 \% \mathrm{CI}-0.001$ to 0.61 ).

Another 2009 meta-analysis of 23 RCTs ( $n=$ 1432) examined the effect of computer-aided psychotherapy on anxiety disorders. ${ }^{11}$ The length of treatment and post-treatment evaluation times varied across the studies: some studies measured outcomes only immediately after treatment, whereas others evaluated outcomes up to one year after treatment. Computer-based CBT showed a large mean effect size of 1.08 (95\% CI 0.84 to 1.32 ) relative to non-computer-based CBT controls, with no difference between faceto-face and computer-based CBT (effect size $-0.06,95 \% \mathrm{CI}-0.22$ to 0.10 ).

A 2007 meta-analysis of 12 RCTs $(n=2334)$ evaluated the effectiveness of Internet-delivered CBT in alleviating depression and anxiety. ${ }^{12}$ Again, length of treatment and post-treatment evaluation times varied. For anxiety, six studies were included, and both the fixed-effects and mixed-effects analyses found reduced symptoms, with an effect size of 0.96 (95\% CI 0.69 to 1.24) for Internet-delivered CBT. When split into unguided and guided Internet-delivered CBT, the effect sizes were 0.24 (95\% CI 0.11 to 0.37 ) and 1.00 (95\% CI 0.75 to 1.24), respectively, which suggests that the guided form is more effective for anxiety disorders than the unguided form. In contrast, the 2009 meta-analysis evaluating depression and anxiety showed no significant differences for patients receiving Internet-delivered CBT with and without therapist contact, ${ }^{8}$ and the 2009 meta-analysis of anxiety disorders showed a small but significant difference in effect size with therapist guidance. ${ }^{11}$

One large cohort study $(n=570)$ evaluated Internet-delivered CBT for panic disorder in routine psychiatric care. ${ }^{13}$ Panic-related anxiety and depression symptoms declined with guided Internet-delivered CBT. Therapist guidance averaged about two hours (total) per patient.

Internet-delivered CBT for anxiety, then, may increase benefits for patients and efficiency for health care professionals, and these data suggest that it may not sacrifice quality of care, despite needing fewer resources.

\section{Depression}

Depression has been the subject of numerous RCTs and subsequent meta-analyses. A 2009 meta-analysis of Internet- and computer-based CBT for adult depression included 12 studies. $^{7}$ The 
depression analysis included 2446 participants, with a pooled effect size of 0.41 (95\% CI 0.29 to 0.54) for Internet-delivered CBT versus all controls. The effect size increased to 0.61 (95\% CI 0.45 to 0.77 ) for therapist-guided studies alone.

The 2007 meta-analysis of Internet-delivered CBT for depression and anxiety, described above, included four RCTs on depression with a total of 1865 participants. ${ }^{12}$ The authors observed reduced burden of depression (effect size $0.22,95 \% \mathrm{CI}$ 0.03 to 0.41 ) with low heterogeneity.

A meta-analysis evaluating Internet- and computer-based interventions in university students was published in $2014 .{ }^{14}$ The length of treatment and post-treatment evaluation times was not assessed. Nine RCTs evaluated depression $(n=712)$, eight were CBT-based $(n=537)$, and only three reported nonskewed outcomes $(n=144)$. The standardized mean difference of the nonskewed data was $-0.67(95 \% \mathrm{CI}-1.15$ to -0.20 ), favouring intervention over nontreatment. When all nine trials were included, the data still favoured Internet-delivered CBT, with a standardized mean difference of -0.43 (95\% CI -0.63 to -0.22 ).

In an RCT comparing Internet-delivered CBT with treatment as usual in 160 young adults, ${ }^{15}$ patients were evaluated at weeks $0,5,10,16$ and 32 after enrolment. A small but significant effect size $(0.20,95 \%$ CI 0.00 to 0.50$)$ was seen in patients who received Internet-delivered CBT relative to controls (treatment as usual), over $50 \%$ of whom had received traditional pharmacotherapy and/or psychotherapy.

The Australia and New Zealand Horizon Scanning Network completed a Prioritization Summary of Internet-delivered CBT for patients with depression, ${ }^{4}$ which included three RCTs and a systematic review evaluating an additional four RCTs. The investigation found that therapistguided Internet-delivered CBT programs achieved substantially higher recovery rates based on the Beck Depression Inventory and the Patient Health Questionnaire Nine Item, and the improved outcomes were sustained for at least eight months. ${ }^{4}$

Overall, the Prioritization Summary ${ }^{4}$ and the three meta-analyses, ${ }^{7,12,14}$ which included a total of 25 RCTs, indicate that Internet-delivered CBT is effective for depression, relative to inactive controls. In addition, the large RCT showed that Internet-based CBT is equivalent to or marginally better than treatment as usual. ${ }^{15}$ These data indicate that Internet-delivered CBT could an effective therapy for depression.

\section{Physical illness}

Internet-delivered CBT for mental health problems has been studied in patients with a range of physical diseases, including diabetes mellitus, ${ }^{16}$ cancer ${ }^{17,18}$ and multiple sclerosis. ${ }^{19}$

One RCT evaluated this form of therapy for depression in 255 patients with diabetes. ${ }^{16}$ The program consisted of eight lessons delivered over eight weeks, and measurements were taken at baseline, immediately after treatment and one month later. In the intention-to-treat analysis, patients who received Internet-delivered CBT showed significant improvements in depression at one-month follow-up compared with waitlisted controls (effect size $0.29,95 \%$ CI 0.17 to 0.40). In the per-protocol analysis, both depression symptoms and diabetes-related distress were reduced from baseline at one month posttreatment compared with wait-listed controls (effect size 0.70 [95\% CI 0.59 to 0.82 ] and 0.58 [95\% CI 0.38 to 0.78], respectively).

Another program, Cancer Coping Online, was developed to target distress among patients with cancer, using six modules delivered weekly. ${ }^{17,18}$ The pilot study, ${ }^{17}$ involving 12 patients evaluated at baseline and seven weeks after initiation of treatment, showed significant reductions in negative affect (effect size 0.53; significance determined by reliable change indices), hopelessness (effect size 0.64) and anxiety (effect size 0.43). Preliminary results of a larger RCT $(n=60)^{18}$ showed significant, sustained interaction effects for cancer-specific distress $\left(F_{1,92}=4.94, p=0.04\right)$ for patients treated with Cancer Coping Online compared with an Internet-attention control (information-only version of the online program, covering the same six module topics but lacking interactive elements).

Recently, researchers developed a fully automated Internet-delivered CBT program called Deprexis to combat depression in patients with multiple sclerosis. ${ }^{19}$ The program included 10 sequential modules, with patients assessed at baseline, immediately after treatment and at six-month follow-up. The effect size for the total Beck Depression Inventory score was $0.53(p=0.01)$ compared with waitlisted controls, based on intention-to-treat analysis $(n=90)$.

Taken together, these studies support the conclusion that Internet-delivered CBT could be an effective treatment for emotional distress caused by medical conditions. In addition, it provides an opportunity for patients with mobility issues to access psychological care.

\section{Special populations}

Several studies have focused on Internetdelivered CBT for special populations, such as children and older adults. Some of these studies are summarized in Box $3 .^{20-25}$ 


\section{Long-term treatment effectiveness}

Most studies of Internet-delivered CBT have limited follow-up (eight weeks to two years). However, one study looked at the efficacy of Internetdelivered CBT for social anxiety disorder over five years. ${ }^{26}$ The program included nine textbased modules. Patients were randomly assigned to treatment and wait-listed control groups. The control group received Internet-delivered CBT after completion of pre- and post-treatment assessments for the experimental group. All patients were included in the one-year and fiveyear follow-up assessments. The authors observed large effect sizes relative to baseline across all

\section{Box 3: Special populations: children and older adults}

\section{Children}

- In several RCTs, juvenile patients with anxiety (aged 8 to 18 years) were responsive to conventional $\mathrm{CBT}$, and the interventions had long-term effectiveness (reviewed by Compton and associates ${ }^{21}$ ).

- A few studies have examined the efficacy of Internet-delivered CBT in children and adolescents. For example, BRAVE-ONLINE and MoodGYM were used in primary care and school-based settings, respectively, for children with anxiety. Both programs were associated with significant improvement in anxiety symptoms (reviewed by Calear and

Christensen ${ }^{22}$ ). Another study ${ }^{20}$ looked at incorporation of MoodGYM into the curriculum in 30 Australian schools. The five-week program was presented by teachers in their own classrooms, and the data were compared with a wait-listed control group who attended unrelated classes. The authors observed significantly greater improvement in anxiety symptoms among participants with high adherence relative to wait-listed controls, both immediately after treatment (effect size 0.34 , $95 \% \mathrm{Cl} 0.11$ to 0.57 ) and at six-month follow-up (effect size $0.39,95 \% \mathrm{Cl}$ 0.16 to 0.62$){ }^{20}$

- Another trial compared the Internet-delivered CBT program Camp CopeA-Lot with individual CBT and computer-linked education, support and attention controls over a 12 -week period. ${ }^{23}$ Both individual CBT and Internet-delivered CBT produced significant improvements relative to controls: $70 \%$ and $81 \%$ of participants, respectively, no longer met their primary diagnostic criteria, compared with $19 \%$ in the control group. There was greater adherence by therapists with the Internet-delivered CBT protocol than with individual CBT, and the patients showed substantially higher satisfaction with Internet-delivered CBT and individual CBT relative to controls.

\section{Older adults}

- It is generally assumed that older adults are reluctant to use new technology and therefore that they would not embrace Internet-delivered CBT. However, several studies evaluating this form of therapy for anxiety have shown that older adults in a primary care setting do well with this intervention. ${ }^{24,25}$

- One open trial determined adherence rates, treatment gains and enrolment patterns for older versus younger adults. ${ }^{24}$ The study used an unguided, six-lesson Internet-delivered CBT program targeting anxiety that was accessible for 90 days. Older patients did not enrol in the Internet-delivered program more frequently than younger adults; however, once enrolled, older patients were substantially more likely to complete the program. There were no significant differences in gains between older adults and younger patients, with one exception. The Mini-Social Phobia Inventory showed greater symptom reduction among older patients than younger patients. The results of this study indicate that Internet-delivered CBT is effective in older populations, but they do not seek it out. Increasing the awareness of available programs could allow use of Internet-delivered CBT to help populations of older adults.

Note: $\mathrm{CBT}=$ cognitive behavioural therapy, $\mathrm{Cl}=$ confidence interval, $\mathrm{RCT}=$ randomized controlled trial. measures, and these were retained for up to five years. The effect sizes at five-year follow-up ranged from 0.63 (95\% CI 0.15 to 1.10$)$ for the Beck Anxiety Inventory and the Quality of Life Inventory scores in the Internet-delivered CBT group to 1.40 (95\% CI 0.86 to 1.90$)$ for the Liebowitz Social Anxiety Scale-Self Report score in the wait-listed control group following administration of Internet-delivered CBT.

There is also some evidence that "booster" sessions (i.e., additional sessions to refresh the principles learned) may be an effective longterm solution. For example, in a recent RCT evaluating booster sessions for patients with obsessive-compulsive disorder, ${ }^{27} 93$ patients received 10 weeks of guided Internet-delivered CBT. Following the post-treatment assessment, they were randomly assigned to booster and nonbooster groups. A three-module weekly booster treatment was administered at six months after completion of initial treatment. All patients were evaluated before treatment, immediately after treatment and at 4, 7, 12 and 24 months. For all groups, there was a large effect size for the primary measure, the YaleBrown Obsessive Compulsive Scale (effect size 2.09 at 24 months compared with pretreatment assessment, $n=87 p<0.001)$. The relapse rate was significantly different between the booster and control groups at the 7- and 12-month assessments, with an estimated risk reduction for relapse of $87 \%(95 \%$ CI $1 \%$ to $98 \%$ ) and $79 \%$ (95\% CI $3 \%$ to $95 \%)$, respectively; however, the groups were not statistically different at 24 months. Secondary measures, including the Global Assessment of Functioning and the Obsessive-Compulsive Inventory - Revised, favoured the booster group, with significant interaction effects retained through 24 months. Because the largest difference in the primary measure was seen at the 7-month assessment, the booster's efficacy may be limited; however, retention of effects for the secondary measures indicates that the booster holds promise, if the treatment protocol can be refined.

\section{What resources are available for Internet-delivered CBT?}

Numerous websites and apps are currently available (Table 1). ${ }^{16-18,24,28-40}$ In Box 3, we have highlighted several programs. An additional program, OCFighter, is described in detail in Box $4 .{ }^{32,33,41}$ In addition, Box 5 outlines the successful rollout by the Australian government of a nationwide Internet-delivered CBT program..$^{24,42}$ 
Table 1: Resources for Internet-delivered cognitive behavioural therapy (CBT) in the literature and the real world

$\begin{array}{lll} & \text { Geographic } \\ \text { Program name } & \text { Description } & \text { availability }\end{array}$

\section{In research trials}

Cancer Coping Online

Available to patients with cancer to help them in coping with distress. The program has 6 modules and takes 6 weeks to complete. High patient satisfaction and relatively good adherence (due to weekly email reminders) was reported. In a pilot trial, 10 of 12 participants completed the full program, and patients stated that the program was easy to use. Patients reported reduction in negative affect, hopelessness and anxiety following completion. ${ }^{17}$ A full clinical trial has recently been completed. ${ }^{18}$

A-CHESS Developed by the Addiction-Comprehensive Health Enhancement Support System as an application (app) that provides support, monitoring and information to patients with alcohol dependence. A-CHESS is part of a behavioural approach for treating this addiction. The app allows patients to stay in contact with counsellors to help maintain sobriety. In an unmasked RCT, use of the app was associated with significant reductions in heavy alcohol consumption over an 8-month period and for 4 months of follow-up. ${ }^{34}$

\section{Currently available*}

THIS WAY UP clinic $†$ (https://thiswayup.org. au/clinic/)

Diabetergestemd.nl (www.diabetergestemd. $\mathrm{nl} /$ ) Spider Phobia Freet and Stress Free (http://virtually-free.com/) Moodkit† (www.thriveport.com/ products/moodkit/) Beating the Bluest (www.beatingtheblues. (o.uk/)

\section{FearFightert}

(www.fearfighter.com/)

\section{CBT-i Coach}

(available on iTunes)

\section{MoodGYM}

(https://moodgym.anu. edu.au/welcome)

Online Therapy USER (www.onlinetherapyuser. ca/wac/welcome-to-thewellbeing-after-cancercourse/)

PTSD Coach Canada (www.veterans.gc.ca/ eng/stay-connected/ canada)

\section{OCFightert}

(www.ocfighter.com/)

\section{Pain Squad}

(www.sickkids.ca/

Research/I-OUCH/

Pain-Squad-App/index. html) mobile-app/ptsd-coach-
Offered to patients with depression and generalized anxiety disorder. Patients must be referred by a physician. The program has been the subject of numerous RCTs. A trajectory study showed significant reductions in psychological distress, measured by the Kessler Psychological Distress Scale (K10), and disability, measured by the World Health Organization Disability Assessment Schedule (WHODAS-II); older adults experienced the most benefit. ${ }^{24,28,30,35}$

Modified version of the Colour Your Life program. Designed to treat depression in patients with type 1 or type 2 diabetes mellitus. An RCT involving 255 adult patients showed reduced emotional distress with no change in glycemic control. ${ }^{16,31}$

Advocated by the NHS and AnxietyUK and designed to provide meditation, relaxation, self-hypnosis and breathing techniques to treat fear, anxiety and stress. An app version of each program is available to Canadians. The UK-based website claims more than 19000 users of the apps.

Designed to provide more than 200 mood-improving activities and thought modulation strategies. Discover magazine called Moodkit "the happiness app."

Recommended by the UK NICE and available in Canada for a fee. The program is for patients with depression or anxiety and consists of eight weekly sessions of 50 minutes each, during which patients are taught to identify symptoms and set goals. Data indicate that statistically significant improvements in key measures of anxiety and depression are achieved. ${ }^{36}$

Designed to treat patients with panic and phobia. This program is available in Canada for a fee; it is covered for NHS patients in the UK. The program consists of nine interactive steps with downloadable content, videos and homework exercises. An RCT for anxiety and depression showed improvement on a number of measures. ${ }^{37}$ Patients and referring health care providers reported a high level of satisfaction and ease with using this program. ${ }^{38}$

Developed by the US Department of Veterans Affairs and designed to help patients with insomnia to improve their sleeping habits and to educate patients on how negative thoughts may affect sleep. In contrast to sleeping medications, Internet-delivered CBT for insomnia focuses on underlying reasons (thoughts) that disrupt or prevent healthy sleep patterns.

Developed in Australia and geared toward patients with anxiety and depression. There are at least 600000 registered users, with translation to Finnish, Dutch, Norwegian and Chinese. The program consists of five interactive modules, quizzes and homework. In an RCT, post-intervention evaluation showed a significantly higher reduction in symptoms among patients who completed the program than among wait-listed controls. ${ }^{29} \mathrm{~A}$ majority of users found this program as acceptable as in-person CBT, stating that they were more likely to continue using MoodGYM over time. ${ }^{29}$

Intended for patients who have recently completed cancer therapy and are experiencing depression or anxiety. The program has five parts and is completed in eight weeks, with weekly email interactions with a therapist. According to the website, 101 patients started the course, and 66 fully completed it; $94 \%$ of those who completed the program felt it was worth their time.

Developed by Veterans Affairs Canada for patients with post-traumatic stress disorder. Focuses on education as well as CBT techniques. The international version of this app (PTSD Coach) is estimated to have been downloaded more than 130000 times in at least 78 countries. In a preliminary evaluation, patients reported satisfaction, improved symptom control and better sleep. ${ }^{39}$

Developed in the UK for patients with OCD, it is available in Canada and the United States for a fee. The program leads to improvement in OCD symptoms. Two RCTs showed good adherence rates with therapist support for as little as 16 minutes per week. ${ }^{32,33}$

Developed by the Hospital for Sick Children (Toronto, Ontario), this app helps in management of pain for children with cancer. A usability and feasibility study showed high compliance rates and overall satisfaction. 40
NA

Australia

Netherlands

Note: INT = internationally available, NA = not applicable, NHS = National Health Service (UK), NICE = National Institute for Health and Care Excellence (UK), $\mathrm{OCD}=$ obsessive-compulsive disorder, $\mathrm{RCT}=$ randomized controlled trial, UK = United Kingdom.

*Additional resources are available, some of which may be found at www.getselfhelp.co.uk/links2.htm.

tFees are applicable for access to these programs. 


\section{What are the advantages of Internet-delivered CBT over face-to-face treatment?}

Internet-delivered CBT has two principle advantages: patient empowerment and increased clinical efficiency.

Patient empowerment has many aspects. With Internet-delivered CBT, patients can schedule their care and receive their therapy from any location with Internet access. ${ }^{7,11}$ This geographic independence benefits patients in rural areas with limited services. For example, one independent study on anxiety evaluated rural populations in Scotland. ${ }^{43}$ Patients participated in FearFighter, a nine-module program, and were assessed before and after treatment and at a follow-up appointment (mean follow-up time 4.5 months, range 1 to 8 months). Among the 35 participants with pretreatment data, $26(74 \%)$ completed treatment. There were moderate to large effect sizes for most anxiety measures, including social anxiety (pre- to post-treatment effect size 0.97; post-treatment to follow-up effect size 0.53 ), depression (pre- to post-treatment effect size 0.99; post-treatment to follow-up effect size 0.03 ) and agoraphobia (pre- to post-treatment effect

\section{Box 4: Featured program - OCFighter}

One example of a well-studied, successful program is OCFighter, an Internetdelivered CBT website for patients with obsessive-compulsive disorder, endorsed by the United Kingdom's National Institute for Health and Care Excellence. This nine-step course features videos of therapists explaining core concepts (such as exposure) and patients discussing their experiences with the illness and their recovery. It is available in Canada and the United States for a fee and includes online therapist support via Skype.

One RCT compared scheduled versus requested therapist support. ${ }^{41}$ Patients had unlimited access to the program for 17 weeks. Those in the scheduled therapist support group completed nine scheduled calls with the therapist, whereas those in the requested therapist support group had access to a help line. Patients were evaluated before and after treatment. The adherence rates for this program were high. Of the scheduled therapist support group ( $n=20$ ), 95\% completed step 7 and $55 \%$ completed the entire program, compared with $57 \%$ and $50 \%$, respectively, for the requested therapist support group $(n=16)$. The scheduled therapist support group showed significant improvements relative to the requested therapist support group, according to scores on the Yale-Brown Obsessive Compulsive Scale (effect size 1.2 v. 0.3 ).

Another RCT compared wait-listed controls with patients receiving OCFighter before face-to-face CBT treatment. ${ }^{32}$ Patients were assessed before and after treatment and also after completing clinician-guided care. The authors observed that the exposure and ritual prevention understanding scores for the OCFighter group were similar to those obtained with in-person CBT (mean score for both 13.1).

A third RCT compared OCFighter with in-person CBT and relaxation controls..$^{33}$ The program was 10 weeks long, and patients were assessed before and after treatment. The results indicated that OCFighter was as effective as in-person CBT if at least one self-exposure session had been completed before initiating Internet-delivered CBT, based on the percentage of responders on the Clinical Global Impressions scale (60\% for in-person CBT v. $65 \%$ for one self-exposure session plus Internet-based CBT).

Note: $\mathrm{CBT}$ = cognitive behavioural therapy, $\mathrm{RCT}$ = randomized controlled trial. size 0.95; post-treatment to follow-up effect size 0.29) ( $p<0.001$ for all comparisons). Thus, Internet-delivered CBT could provide care for patients who might not seek treatment because of physical or psychiatric conditions or social stigma. Several recent clinical trials implemented self-referral as a recruitment option. ${ }^{20,28,43,44}$ In addition, the privacy and anonymity of Internet-delivered CBT may help patients who are introverted or depressed to be more open and receptive to treatment. ${ }^{7}$

From a clinical efficiency perspective, Internetdelivered CBT offers substantial benefits. It allows clinicians to treat more patients effectively in less time. Even with intermittent therapist support, it is less time-consuming and requires fewer resources overall than traditional CBT. For example, a 2014 editorial about guided Internet-delivered CBT noted that therapists spent about 10 minutes per patient per week, ${ }^{45}$ and in one RCT investigating Internet-delivered CBT for irritable bowel syndrome, six therapists treated 195 patients simultaneously. ${ }^{46}$ In addition, a 2009 review of what makes Internet-delivered CBT effective stated that asynchronous therapist communications are advantageous because they minimize the need for immediate availability. ${ }^{7}$ A 2014 review discussing the advantages and limitations of Internet-delivered CBT noted that the incorporation of questionnaires, homework and self-assessments allowed the patient's progress and outcome to be easily monitored. ${ }^{3}$

\section{Is Internet-delivered CBT cost-effective?}

Several studies have investigated the cost-effectiveness of Internet-delivered CBT from both societal and provider perspectives. In a 2012 systematic review of this modality and its applications, efficacy and cost-effectiveness, the authors calculated that the probability of Internet-delivered CBT being a cost-effective treatment was 57\% (range 38\% to 96\%) relative to wait-listed controls. ${ }^{6}$ One RCT compared in-person group CBT with Internetdelivered CBT for social anxiety. ${ }^{47}$ Patients were treated through a 15 -module Internet-delivered program or 14 weekly group meetings and were assessed before treatment, immediately after treatment and six months after treatment. The authors determined that the probability of Internetdelivered CBT being efficacious at a lower cost was $79.5 \%$ at a willingness-to-pay of US\$0. Two studies compared a 10-module Internet-delivered CBT program with a stress-management strategy ${ }^{46}$ or an Internet chat forum ${ }^{48}$ for irritable bowel syndrome. The authors calculated a societal cost reduction of about US\$16000 and US\$39000 per patient compared with forum and stress-manage- 
ment controls, respectively, at a willingness-to-pay of US\$0. Another RCT compared a four-month, 10-module Internet-delivered CBT program with treatment as usual for depression. ${ }^{49}$ Using data from this RCT, a systematic review calculated a probability of about $70 \%$ that Internet-delivered CBT was cost-effective at a willingness-to-pay of US\$50 000.50 Two RCTs surveyed guided Internet-delivered CBT for depression ${ }^{51}$ and anxiety ${ }^{52}$ in older adults. Both programs were five-lesson, eight-week courses, and data were compared with data for wait-listed control groups. The authors showed that both programs had a greater than $95 \%$ chance of being cost-effective at the standard Australian willingness-to-pay threshold of A\$50 000.

All of these studies calculated the cost-effectiveness of pre-existing, successful programs, but developing a new program requires substantial time and money. Of course, it is less expensive to translate an existing program to a new language. To assess the cost-effectiveness of translation, researchers translated the Internet-delivered CBT programs BluePages and MoodGYM from English into Norwegian and conducted an RCT comparing the participants in the translated programs with wait-listed controls. ${ }^{53}$ The cost of translation was about one-fourth the cost of a new program, and program implementation would produce a 21-fold return on investment based on the qualityadjusted life-years gained. These data support the translation of existing programs, rather than investment in the development of new programs.

\section{What are the disadvantages of Internet-delivered CBT?}

There are some disadvantages to Internet delivery of CBT. The first is lack of direct patient monitoring, which can be problematic for two reasons: lack of a human relationship (i.e., therapeutic alliance) and difficulty in adjusting therapy according to the patient's progress. A few studies, including one systematic review, have evaluated therapeutic alliance in guided Internet-delivered CBT for several conditions, mainly compared with wait-listed controls or email-based therapist interaction. ${ }^{54-59}$ These studies consistently showed high therapeutic alliance for guided Internet-delivered CBT. Only one RCT directly compared guided with face-to-face Internetdelivered CBT. ${ }^{60}$ The study enrolled 86 patients with panic disorder, divided into two groups: the Internet-delivered CBT group received the sixmodule Panic Online program, and the face-toface group received weekly one-hour sessions for 12 weeks. The authors observed no difference in outcomes or therapeutic alliance scores between the two groups. However, patients in the face-to- face group reported greater enjoyment communicating with their therapist. ${ }^{60}$ The difficulty of adjusting a patient's therapy can be mitigated somewhat by using guided Internet-delivered CBT, whereby a therapist assesses the patient's progress and adapts treatment accordingly. ${ }^{3}$

The second disadvantage is low adherence. Several studies indicate that this issue may be mitigated by some level of therapist support. For example, in an RCT comparing the effectiveness of the MoodGYM program with wait-listed controls, ${ }^{29} 73 \%$ of unguided MoodGYM users did not complete the full program. In contrast, therapistguided MoodGYM users had completion rates equal to those for traditional CBT. ${ }^{29}$ Another RCT evaluated various levels of therapist support with the Panic Online program. ${ }^{61}$ The patients were assigned to groups with frequent or infrequent therapist contact, defined as receiving at least three or just one email message per week, respectively. The researchers observed no differences between the two groups in efficacy, therapeutic alliance, treatment credibility, treatment satisfaction or treatment adherence. ${ }^{61}$ In addition, a series of studies using the Internet-delivered Worry program to treat anxiety showed that therapy guided by primary care physicians achieved $40 \%$ to $60 \%$ adherence, and therapy guided by clinical psychologists had rates as high as $90 \% .^{28,30,62} \mathrm{In}$ contrast, one RCT directly compared unguided, minimally guided and flexibly guided Internetdelivered CBT for social phobias using a fivelesson program administered over 10 weeks. ${ }^{63}$ The three treatments were equally effective, with no statistical difference in adherence. Further studies are needed to reconcile these conflicting results.

\section{Box 5: Example of government rollout - Australia}

Several clinical Internet-delivered CBT programs have already been rolled out successfully. Governments are embracing websites and apps, including the United Kingdom (England and Wales), Sweden, Australia and Canada (Saskatchewan). One example is Australia's THIS WAY UP Clinic. This e-clinic, the result of a university-state government partnership, offers a number of courses: Worry (for generalized anxiety disorder), Worry and Sadness (for mixed depression and anxiety), Shyness (for social phobias), Obsessions and Compulsions (for obsessive-compulsive disorder) and Panic (for panic and agoraphobia). The THIS WAY UP Clinic makes good use of the Internet: patients can set up an online calendar to generate email reminders, progress reports are provided to referring clinicians by email, patients have access to "patient recovery stories," and the website is graphically pleasing and uses comic book-style lessons to explain key concepts in story form.

The courses are available by referral through clinic-registered clinicians, including family doctors but also mental health nurses and psychologists. The program charges a fee $(A \$ 55)$. The e-clinic also features free self-help courses. A recent progress update by the e-Mental Health Alliance reported that the THIS WAY UP Clinic was logging an average of 1000 unique visitors and 80 patients registering per month. ${ }^{42}$ Newby and associates ${ }^{24}$ reported that more than 3600 clinicians were registered with THIS WAY UP as of May 2013. The self-help program alone had over 6000 registrations since its inception and about 275 registrations per month.

Note: $\mathrm{CBT}=$ cognitive behavioural therapy. 
The third disadvantage of Internet-delivered CBT relates to technology accessibility. As much as 30\% of the Western population may not have home Internet access or own a computer. ${ }^{7}$ In addition, the programs may have hardware or bandwidth requirements that limit their availability to patients. For example, for RCTs of Internet-delivered CBT in rural Scotland and China, computers had to be provided or computer centres created to allow patients to complete the program..$^{43,64}$ In addition, patients who are not technologically savvy may resist replacing clinical visits with computerized treatments.

The fourth disadvantage is the possibility of improper treatment. Internet-delivered CBT occasionally uses online diagnostic tools based on self-reporting, which could lead to misdiagnosis and improper treatment. One study compared an Internet-administered Composite International Diagnostic Interview with an in-person Structured Clinical Interview for DSM-IV [Diagnostic and Statistical Manual of Mental Disorders, Fourth Edition] axis I disorders. ${ }^{65}$ Among 53 patients who performed an online diagnostic interview two days before a formal in-person interview, there was low agreement between the two evaluation methods for all but panic disorder. In contrast, a recent study comparing automated pretreatment assessments $(n=173)$ with clinician diagnoses $(n=135)$ for assignment to an Internetdelivered CBT program ${ }^{66}$ showed similar results in the two groups.

There are also potential concerns regarding service providers and their products, such as poor program quality or fake services. So far, no one has investigated these concerns. However, users can apply some common sense precautions in selecting a program. Legitimate programs often have government endorsements, are affiliated with a hospital or academic institution, or feature relevant peer-reviewed literature. For programs with therapist guidance, the therapists' biographies are frequently available, and the patient can check their credentials. These features can help in distinguishing legitimate programs from scams. However, a patient who feels that a service has

\section{Box 6: Questions for future research}

- What types of patients would benefit from Internet-delivered cognitive behavioural therapy (CBT)?

- Is Internet-delivered CBT truly as effective as in-person CBT?

- How should Internet-delivered CBT be blended with other therapies, such as pharmacotherapy or in-person support?

- How can Internet-delivered CBT be implemented in the clinic?

- How can Internet-delivered CBT be scaled up to reach those who need it most? not delivered what was advertised has limited options for redress. If a well-researched, government-supported program was used, a complaint can be filed with the organization's customer service department. Some commercial apps have customer service departments as well. In either case, the best outcome is likely to be a refund. Government programs are more likely to be responsive to complaints, in an effort to maintain their funding. Although suppliers of commercial apps may be less likely to respond to complaints, most of these apps cost less than US $\$ 5$, so the monetary loss will be minimal.

\section{Who should not pursue Internet- delivered CBT?}

Internet-delivered CBT is not recommended for severely ill patients. In fact, most studies have excluded severely ill patients, on the assumption that this form of therapy is better suited for patients with early-stage disease and sufficient social support. ${ }^{67}$ However, a secondary analysis of data from the diabetes RCT, described above, ${ }^{16}$ showed that symptom severity was not a significant effect modifier for success. ${ }^{31}$ As part of a quality assurance study, researchers followed the outcomes for 359 patients for whom the Australian Sadness program had been prescribed ${ }^{68}$ and observed large post-treatment effect sizes in patients with severe symptoms or suicidal tendencies (Patient Health Questionnaire Nine Item: effect size 1.49, 95\% CI 1.01 to 1.96; Patient Health Questionnaire Nine Item - Suicide Item: effect size 1.12, 95\% CI 0.64 to 1.59 ). Further studies are needed to assess the effectiveness of Internet-delivered CBT for severe illness, but substantial ethical concerns related to trial design make such studies challenging.

\section{What are the future directions, challenges and obstacles for integration into clinical practice?}

Although a growing body of research favours Internet-delivered CBT, some important questions must be addressed (Box 6). First, what types of patients will benefit from this form of therapy? Currently, there is no clear picture of the ideal demographic. ${ }^{3}$ The applicability and benefits of Internet-delivered CBT for specific populations, including economically disadvantaged and poorly educated people, as well as those living in rural areas, need further investigation. ${ }^{68}$ Second, is Internet-delivered CBT truly as effective as in-person CBT? Given the limited number of studies directly comparing inperson and Internet-delivered CBT, ${ }^{9}$ future trials should involve head-to-head comparisons. 
The implementation of Internet-delivered CBT also poses several clinical challenges. The first is that of blending this form of CBT with other therapies, such as pharmacotherapy or in-person support. Further studies are needed to identify the most effective combination of Internet-delivered CBT and other treatments. The second challenge relates to clinical implementation. The number of physicians available for guided Internet-delivered CBT is limited. However, a recent RCT on generalized anxiety disorder compared the efficacy of guidance by clinicians and nonpsychologist technicians. ${ }^{69}$ Patients were divided into three groups: clinician-guided Internet-delivered CBT, technician-guided Internet-delivered CBT and waitlisted controls. The two Internet-delivered CBT groups had access to the Worry program (six modules, 10 weeks) and were evaluated before and immediately after treatment and at threemonth follow-up. The technician-guided and clinician-guided Internet-delivered CBT groups were similar in terms of adherence (80\% v. $74 \%)$ and outcomes (Penn State Worry Questionnaire pre-treatment to follow-up effect size 0.97 v. 1.42; Generalized Anxiety Disorder Seven-Item effect size $1.61 \mathrm{v} .1 .48$ ). These data showed that a modestly trained individual with no prior counselling qualifications could guide Internet-delivered CBT, with adherence and outcomes similar to those achieved with clinician guidance, indicating that well-trained assistants may be used to supplement clinicians. The third challenge is scale-up. Several large-scale government rollouts have supported Internet-delivered CBT as a feasible national health care option. The e-Mental Health Alliance in Australia has outlined its own challenges for scaling up Internet-delivered CBT, specifically increasing awareness of available services and optimizing the programs to increase adherence and maximize outcomes. ${ }^{42}$ These factors should be considered in future large-scale investigations.

\section{Conclusion}

Overall, there are compelling data to support the integration of Internet-delivered CBT into clinical psychiatric care. These data indicate that this form of CBT offers numerous benefits to both the patient and the practitioner. It allows treatment of patients with many different psychiatric conditions, at lower cost than traditional CBT. Patient adherence can be improved with some therapist involvement, and the evidence suggests that this approach yields better outcomes. Additionally, governments are likely to find this treatment modality attractive in terms of cost and efficacy. In the age of Google, this psychological intervention is empowering, clinically efficient and consis- tent with the way that, increasingly, patients interact with health care.

\section{References}

1. Ravindran AV, Lam RW, Filteau MJ, et al. Canadian Network for Mood and Anxiety Treatments (CANMAT) clinical guidelines for the management of major depressive disorder in adults: V. Complementary and alternative medicine treatments. $J$ Affect Disord 2009;117 Suppl 1:S54-64.

2. Brewin CR. Theoretical foundations of cognitive-behavior therapy for anxiety and depression. Annu Rev Psychol 1996;47:33-57.

3. Andersson G, Titov N. Advantages and limitations of Internetbased interventions for common mental disorders. World Psychiatry 2014;13:4-11.

4. Australia and New Zealand Health Scanning Network. Horizon scanning technology prioritising summary: Internet-delivered cognitive behavioural therapy for patients with depression. Canberra: Commonwealth of Australia Department of Health and Ageing; 2009.

5. Online therapy user update. Regina: University of Regina; 2013.

6. Hedman E, Ljotsson B, Lindefors N. Cognitive behavior therapy via the Internet: a systematic review of applications, clinical efficacy and cost-effectiveness. Expert Rev Pharmacoecon Outcomes Res 2012;12:745-64.

7. Andersson G, Cuijpers P. Internet-based and other computerized psychological treatments for adult depression: a meta-analysis. Cogn Behav Ther 2009;38:196-205.

8. Reger MA, Gahm GA. A meta-analysis of the effects of internet- and computer-based cognitive-behavioral treatments for anxiety. J Clin Psychol 2009;65:53-75.

9. van Ballegooijen W, Cuijpers P, van Straten A, et al. Adherence to Internet-based and face-to-face cognitive behavioural therapy for depression: a meta-analysis. PLoS One 2014;9:e100674.

10. Cohen, J. A power primer. Psychol Bull 1992;112:155-9.

11. Cuijpers P, Marks IM, van Straten A, et al. Computer-aided psychotherapy for anxiety disorders: a meta-analytic review. Cogn Behav Ther 2009;38:66-82.

12. Spek V, Cuijpers P, Nyklicek I, et al. Internet-based cognitive behaviour therapy for symptoms of depression and anxiety: a meta-analysis. Psychol Med 2007;37:319-28.

13. Hedman E, Ljotsson B, Ruck C, et al. Effectiveness of internetbased cognitive behaviour therapy for panic disorder in routine psychiatric care. Acta Psychiatr Scand 2013;128:457-67.

14. Davies EB, Morriss R, Glazebrook C. Computer-delivered and Web-based interventions to improve depression, anxiety, and psychological well-being of university students: a systematic review and meta-analysis. J Med Internet Res 2014;16:e130.

15. Clarke G, Kelleher C, Hornbrook M, et al. Randomized effectiveness trial of an Internet, pure self-help, cognitive behavioral intervention for depressive symptoms in young adults. Cogn Behav Ther 2009;38:222-34.

16. van Bastelaar KM, Pouwer F, Cuijpers P, et al. Web-based depression treatment for type 1 and type 2 diabetic patients: a randomized, controlled trial. Diabetes Care 2011;34:320-5.

17. Beatty LJ, Koczwara B, Wade TD. 'Cancer Coping Online': a pilot trial of a self-guided CBT internet intervention for cancerrelated distress. Electron J Appl Psychol 2011;7:17-25.

18. Beatty L, Koczwara B, Wade T. Cancer Coping Online: findings and lessons learned from a phase II RCT of an eHealth program for reducing cancer-distress [poster abstract]. In: Proceedings of the Clinical Oncological Society of Australia Annual Scientific Meeting; 2013 Nov. 12-14; Adelaide, Australia. Asia Pac J Clin Oncol 2013;9(S3 Suppl):48-59.

19. Fischer A, Schröder J, Vettorazzi E, et al. An online programme to reduce depression in patients with multiple sclerosis: a randomised controlled trial. Lancet Psychiatry 2015;2:217-23.

20. Calear AL, Christensen H, Mackinnon A, et al. Adherence to the MoodGYM program: outcomes and predictors for an adolescent school-based population. J Affect Disord 2013;147:338-44.

21. Compton SN, March JS, Brent D, et al. Cognitive-behavioral psychotherapy for anxiety and depressive disorders in children and adolescents: an evidence-based medicine review. J Am Acad Child Adolesc Psychiatry 2004;43:930-59.

22. Calear AL, Christensen H. Review of internet-based prevention and treatment programs for anxiety and depression in children and adolescents. Med J Aust 2010;192(11 Suppl):S12-4.

23. Khanna MS, Kendall PC. Computer-assisted cognitive behavioral therapy for child anxiety: results of a randomized clinical trial. J Consult Clin Psychol 2010;78:737-45.

24. Newby JM, Mewton L, Williams AD, et al. Effectiveness of transdiagnostic Internet cognitive behavioural treatment for mixed anxiety and depression in primary care. J Affect Disord 
2014;165:45-52.

25. Mewton L, Sachdev PS, Andrews G. A naturalistic study of the acceptability and effectiveness of internet-delivered cognitive behavioural therapy for psychiatric disorders in older Australians. PLoS One 2013;8:e71825.

26. Hedman E, Furmark T, Carlbring P, et al. A 5-year follow-up of Internet-based cognitive behavior therapy for social anxiety disorder. J Med Internet Res 2011;13:e39.

27. Andersson E, Steneby S, Karlsson K, et al. Long-term efficacy of Internet-based cognitive behavior therapy for obsessivecompulsive disorder with or without booster: a randomized controlled trial. Psychol Med 2014;44:2877-87.

28. Williams AD, O'Moore K, Mason E, et al. The effectiveness of internet cognitive behaviour therapy (iCBT) for social anxiety disorder across two routine practice pathways. Internet Interv 2014; 1:225-9.

29. Twomey C, O'Reilly G, Byrne M, et al. A randomized controlled trial of the computerized CBT programme, MoodGYM, for public mental health service users waiting for interventions. Br J Clin Psychol 2014;53:433-50.

30. Newby JM, Mackenzie A, Williams AD, et al. Internet cognitive behavioural therapy for mixed anxiety and depression: a randomized controlled trial and evidence of effectiveness in primary care. Psychol Med 2013;43:2635-48.

31. van Bastelaar KM, Pouwer F, Cuijpers P, et al. Is a severe clinical profile an effect modifier in a Web-based depression treatment for adults with type 1 or type 2 diabetes? Secondary analyses from a randomized controlled trial. J Med Internet Res 2012;14:e2.

32. Nakagawa A, Marks IM, Park JM, et al. Self-treatment of obsessive-compulsive disorder guided by manual and computerconducted telephone interview. J Telemed Telecare 2000;6:22-6.

33. Greist JH, Marks IM, Baer L, et al. Behavior therapy for obsessive-compulsive disorder guided by a computer or by a clinician compared with relaxation as a control. J Clin Psychiatry 2002; 63:138-45.

34. Gustafson DH, McTavish FM, Chih M, et al. A smartphone application to support recovery from alcoholism: a randomized clinical trial. JAMA Psychiatry 2014;71:566-72.

35. Sunderland M, Wong N, Hilvert-Bruce Z, et al. Investigating trajectories of change in psychological distress amongst patients with depression and generalised anxiety disorder treated with internet cognitive behavioural therapy. Behav Res Ther 2012;50:374-80.

36. Cavanagh K, Seccombe N, Lidbetter N. The implementation of computerized cognitive behavioural therapies in a service userled, third sector self-help clinic. Behav Cogn Psychother 2011; 39:427-42.

37. Proudfoot J, Ryden C, Everitt B, et al. Clinical efficacy of com puterised cognitive-behavioural therapy for anxiety and depression in primary care: randomised controlled trial. $\mathrm{Br} \mathrm{J}$ Psychiatry 2004;185:46-54.

38. MacGregor AD, Hayward L, Peck DF, et al. Empirically grounded clinical interventions clients' and referrers' perceptions of computer-guided CBT (FearFighter). Behav Cogn Psychother 2009;37:1-9.

39. Kuhn E, Greene C, Hoffman J, et al. Preliminary evaluation of PTSD Coach, a smartphone app for post-traumatic stress symptoms. Mil Med 2014;179:12-8.

40. Stinson JN, Jibb LA, Nguyen C, et al. Development and testing of a multidimensional iPhone pain assessment application for adolescents with cancer. J Med Internet Res 2013;15:e51.

41. Kenwright M, Marks I, Graham C, et al. Brief scheduled phone support from a clinician to enhance computer-aided self-help for obsessive-compulsive disorder: randomized controlled trial. J Clin Psychol 2005;61:1499-508.

42. Christensen H, Proudfoot J, Woodward A, et al. e-Mental Health services in Australia 2014: current and future. Australia: e-Mental Health Alliance; 2014. 28 p. Available: https://emhalliance. fedehealth.org au/wp-content/uploads/sites/42/2014/10/e-Mental -Health-in-Australia-2014.pdf (accessed 2015 Jan. 8).

43. Hayward L, MacGregor AD, Peck DF, et al. The feasibility and effectiveness of computer-guided CBT (FearFighter) in a rural area. Behav Cogn Psychother 2007;35:409-19.

44. Dear BF, Titov N, Perry KN, et al. The Pain Course: a randomised controlled trial of a clinician-guided Internet-delivered cognitive behaviour therapy program for managing chronic pain and emotional well-being. Pain 2013;154:942-50

45. Hedman E. Therapist-guided Internet-delivered cognitive behavioural therapy [editorial]. BMJ 2014;348:g1977.

46. Ljótsson B, Hedman E, Andersson E, et al. Internet-delivered exposure-based treatment vs. stress management for irritable bowel syndrome: a randomized trial. Am J Gastroenterol 2011;106:1481-91.

47. Hedman E, Andersson E, Ljotsson B, et al. Cost-effectiveness of Internet-based cognitive behavior therapy vs. cognitive behavioral group therapy for social anxiety disorder: results from randomized controlled trial. Behav Res Ther 2011;49:729-36.
48. Andersson E, Ljotsson B, Smit F, et al. Cost-effectiveness of internet-based cognitive behavior therapy for irritable bowel syndrome: results from a randomized controlled trial. BMC Public Health 2011;11:215.

49. Hollinghurst S, Peters TJ, Kaur S, et al. Cost-effectiveness of therapist-delivered online cognitive-behavioural therapy for depression: randomised controlled trial. Br J Psychiatry 2010; 197:297-304

50. Arnberg FK, Linton SJ, Hultcrantz M, et al. Internet-delivered psychological treatments for mood and anxiety disorders: a systematic review of their efficacy, safety, and cost-effectiveness. PLoS One 2014;9:e98118.

51. Titov N, Dear BF, Ali S, et al. Clinical and cost-effectiveness of therapist-guided Internet-delivered cognitive behavior therapy for older adults with symptoms of depression: a randomized controlled trial. Behav Ther 2015;46:193-205.

52. Dear BF, Zou JB, Ali S, et al. Clinical and cost-effectiveness of therapist-guided Internet-delivered cognitive behavior therapy for older adults with symptoms of anxiety: a randomized controlled trial. Behav Ther 2015;46:206-17.

53. Lintvedt OK, Griffiths KM, Eisemann M, et al. Evaluating the translation process of an Internet-based self-help intervention for prevention of depression: a cost-effectiveness analysis. $\mathrm{J} \mathrm{Med}$ Internet Res 2013;15:e18.

54. Sucala M, Schnur JB, Constantino MJ, et al. The therapeutic relationship in e-therapy for mental health: a systematic review. J Med Internet Res 2012;14:e110.

55. Andersson G, Paxling B, Wiwe M, et al. Therapeutic alliance in guided Internet-delivered cognitive behavioural treatment of depression, generalized anxiety disorder and social anxiety disorder. Behav Res Ther 2012;50:544-50.

56. Knaevelsrud C, Maercker A. Does the quality of the working alliance predict treatment outcome in online psychotherapy for traumatized patients? J Med Internet Res 2006;8:e31.

57. Knaevelsrud C, Maercker A. Internet-based treatment for PTSD reduces distress and facilitates the development of a strong therapeutic alliance: a randomized controlled clinical trial. $B M C$ Psychiatry 2007;7:13.

58. Ruwaard J, Lange A, Bouwman M, et al. E-mailed standardized cognitive behavioural treatment of work-related stress: a randomized controlled trial. Cogn Behav Ther 2007;36:179-92.

59. Ruwaard J, Schrieken B, Schrijver M, et al. Standardized webbased cognitive behavioural therapy of mild to moderate depression: a randomized controlled trial with a long-term follow-up. Cogn Behav Ther 2009;38:206-21.

60. Kiropoulos LA, Klein B, Austin DW, et al. Is Internet-based CBT for panic disorder and agoraphobia as effective as face-toface CBT? J Anxiety Disord 2008;22:1273-84.

61. Klein B, Austin D, Pier C, et al. Internet-based treatment for panic disorder: Does frequency of therapist contact make a difference? Cogn Behav Ther 2009;38:100-13.

62. Mewton L, Smith J, Rossouw P, et al. Current perspectives on Internet-delivered cognitive behavioral therapy for adults with anxiety and related disorders. Psychol Res Behav Manag 2014 7:37-46.

63. Berger T, Caspar F, Richardson R, et al. Internet-based treatment of social phobia: a randomized controlled trial comparing unguided with two types of guided self-help. Behav Res Ther 2011;49:158-69.

64. Wang Z, Wang J, Maercker A. Chinese My Trauma Recovery, a Web-based intervention for traumatized persons in two paralle samples: randomized controlled trial. J Med Internet Res 2013; 15:e213.

65. Carlbring $\mathrm{P}$, Forslin $\mathrm{P}$, Ljungstrand $\mathrm{P}$, et al. Is the Internetadministered CIDI-SF equivalent to a clinician-administered SCID interview? Cogn Behav Ther 2002;31:183-9.

66. Mason EC, Andrews G. The use of automated assessments in internet-based CBT: the computer will be with you shortly. Internet Intervention 2014;1:216-24.

67. Carlbring P, Andersson G. Internet and psychological treatment How well can they be combined? Comput Human Behav 2006; 22:545-53.

68. Williams AD, Andrews G. The effectiveness of Internet cognitive behavioural therapy (iCBT) for depression in primary care: a quality assurance study. PLoS One 2013;8:e57447.

69. Robinson E, Titov N, Andrews G, et al. Internet treatment for generalized anxiety disorder: a randomized controlled trial comparing clinician vs. technician assistance. PLoS One 2010;5:e10942.

Affiliation: The Scarborough Hospital, Toronto, Ont.

Contributors: Both authors participated in conceiving the article, searching the literature, analyzing and interpreting the results of the search, and writing and editing the manuscript, and both agree to act as guarantors of the work. 